\title{
Effet Auger généré par rayons $X$ issus de l'explosion d'agrégats soumis à un champ laser intense
}

\author{
P. Palianov, J. Gaudin, P. Martin, H. Merdji , P. Meynadier ${ }^{1}$ et M. Perdrix ${ }^{1}$ \\ CEA-DSM/DRECAM/LSI, École Polytechnique, 91128 Palaiseau, France \\ ${ }^{1}$ CEA-DSM/DRECAM/SPAM, CE-Saclay, 91191 Gif-sur-Yvette cedex, France
}

\begin{abstract}
Résumé : Nous avons réalisé des expériences portant sur la caractérisation du signal de photoélectrons d'une cible de cuivre pure irradiée par les rayons $X$ issus de l'interaction d'un laser femtoseconde Ti:Sa avec un jet d'agrégats de gaz rares ( $\mathrm{Ar}, \mathrm{Kr}$ et $\mathrm{Xe}$ ). Nous avons étudié l'influence de différents paramètres: type de gaz, pressions du jet, durée d'impulsion et longueur d'onde du laser (fondamental à $800 \mathrm{~nm}$ et seconde harmonique à $400 \mathrm{~nm}$ ) sur l'amplitude du signal obtenu. Nos expériences ont permis de mettre en évidence les meilleurs conditions : 30 bars de xénon irradié par des impulsions de $250 \mathrm{fs}$ à $800 \mathrm{~nm}$ conduisant à un éclairement d'environ $2.10^{16} \mathrm{~W} / \mathrm{cm}^{2}$. Dans ces conditions nous avons pu mesurer un pic d'électron Auger situé à $62 \mathrm{eV}$. Dès lors nous pensons qu'il est possible de mesurer la durée de l'impulsion $\mathrm{X}$ en utilisant une méthode d'intercorrélation basée sur l'effet Auger ( Laser Assisted Auger Decay $n$ ).
\end{abstract}

Les sources de rayonnement brillantes et compactes dans le domaines UVX font actuellement l'objet de recherches intensives vu leur fort potentiel d'application aussi bien dans l'industrie que dans la recherche. Une des configurations les plus avantageuses pour obtenir une source de rayons $\mathrm{X}$ pulsée dans le domaine $\mathrm{du} \mathrm{keV}$ s'est avérée être celle obtenue par l'interaction d'agrégats de gaz rare avec une impulsion sub-picoseconde. Cette architecture donne un bon rendement (comparée aux cibles gazeuses) et ne produit pas de débris lors de l'interaction (contrairement aux cibles solides). Les recherches menées sur ce type de source ont permis de mieux comprendre les mécanismes d'émission (modèle des nano-plasmas[1]) et de caractériser leur spectre[2] ainsi que les particules émises : ions multichargés [3] et électrons énergétiques [4]. Cependant la durée des impulsions obtenues reste sujet à controverses [5]. Une méthode possible [6] de mesure de durée d'impulsion consiste à soumettre une cible au flux de photons X. Des électrons sont alors émis par effet Auger[7]. Ils sont identifiables dans le spectre de photoélectrons par la présence de pics à des énergies caractéristiques indépendantes de la source d'excitation. Si dans le même temps la cible est irradiée par une impulsion laser les électrons éjectés vont absorber ou émettre des photons. Le spectre de photoélectrons est alors modifié : des pics satellites vont apparaître séparés par un quantum d'énergie du laser. En décalant dans le temps l'impulsion laser par apport à l'impulsion X l'intensité des pics du spectre varie. Le signal de photoémission est alors proportionnel au signal d'intercorrélation[8] des deux impulsions. Ainsi partant d'une impulsion laser dont la durée est connue il devient possible de calculer la durée de l'impulsion X. Cependant la probabilité d'émission d'un électron Auger est faible. Il convient donc pour obtenir un signal exploitable d'optimiser au maximum les paramètres expérimentaux. 


\section{MONTAGE EXPERIMENTAL}

Le laser utilisé est un système Ti :Sa délivrant des impulsions infrarouges, centrées sur 800 $\mathrm{nm}$, de durée typique de $50 \mathrm{fs}$ à quelques ps jusqu'à des énergies de $70 \mathrm{~mJ}$ à une fréquence de $20 \mathrm{~Hz}$.

Le faisceau est focalisé par une lentille ( $f=500 \mathrm{~mm}$ ) sur le jet de gaz permettant d'obtenir des éclairements de l'ordre de $10^{17} \mathrm{~W} / \mathrm{cm}^{2}$ pour $60 \mathrm{fs}$. Le diamètre de la vanne d'éjection est de $300 \mu \mathrm{m}$. La buse qui permet la détente est un cône d'angle $7.5^{\circ}$ et de hauteur égale à 25 $\mathrm{mm}$. La zone d'interaction où sont produits les rayons $X$ se situe $5 \mathrm{~mm}$ sous la sortie de la buse. La pression dans le jet peut aller jusqu'à 60 bars dans un vide environnant de $10^{-6}$ mbar. Les agrégats sont produits lors d'une détente adiabatique. Leur taille varie entre 100 et $300 \AA$ avec de $10^{4}$ à $10^{7}$ atomes/agrégats[9] .

Un filtre en béryllium (épaisseur $30 \mu \mathrm{m}$ ) est placé entre la source $X$ et l'échantillon afin de stopper les électrons et les ions émis ainsi que le rayonnement UV. Le faisceau $x$ arrive sous un angle de $60^{\circ}$ sur la cible. Le spectromètre utilisé est un system CLAM 4 VG Microtech. La cible de cuivre pur est nettoyée par bombardement ionique avec de l'argon.

La géométrie du dispositif expérimental permet de collecter au maximum $0.1 \%$ des $10^{5}$ à $10^{7}$ photons émis par tir laser (l'émission est isotrope sur $4 \pi \mathrm{sr}$, alors que l'angle solide correspondant aux photons arrivant sur la cible est de $2.10^{-2} \mathrm{sr}$ ). L'optimisation du signal par le choix de paramètres expérimentaux adéquats est donc primordiale.

\section{RESULTATS EXPERIMENTAUX}

\subsection{Optimisation du signal de photoémission mesuré}

Pour des paramètres laser $\left(800 \mathrm{~nm}, 60 \mathrm{fs}, 10^{17} \mathrm{~W} / \mathrm{cm}^{2}\right)$ et pression (jet de 40 bars) similaires nous avons mesuré le signal de photoémission obtenu avec du krypton de l'argon et du xénon. Le signal issu du Xe est supérieur d'un ordre de grandeur à celui obtenu pour $\mathrm{Kr}$, et supérieur de deux ordres de grandeur à celui obtenu avec Ar. Cela peut sembler surprenant aux vues des résultats obtenus par Rozet et al. [2] montrant le taux de production absolu $(\mathrm{N}=$ nombre de photon/tir laser) pour les raies à $1741 / 2248 \mathrm{eV}$ du krypon $\mathrm{N}=4.10^{6}$, à $4109 \mathrm{eV}$ du xénon $\mathrm{N}=1,5.10^{5}$ et à $3086 \mathrm{eV}$ de l'argon $\mathrm{N}=7.10^{4}$. Nos expériences ne sont pas sélectives en longueur d'onde ce qui explique cet apparent désaccord. En effet le signal de photoélectrons est proportionnel au spectre intégré sur le domaine où l'énergie des photons est supérieure à celle de l'énergie de liaison de l'électron. Le maximum de signal de photoélectron ne dépend donc pas de l'intensité d'une raie donnée, mais de tout le spectre intégré. Dans la suite de nos expériences nous avons donc utilisé le xénon.

L'influence de la pression a été étudiée conjointement pour 800 et $400 \mathrm{~nm}$ pour le xénon. Pour le fondamental le signal est proportionnel à $\mathrm{P}^{5 / 3}$ ( $\mathrm{P}$ :pression du jet de gaz) comme attendu [2] alors que pour la seconde harmonique le signal évolue de façon quasi linéaire avec la pression. Comme attendu [10] le signal dépend de la durée d'impulsion. Nous avons trouvé un maximum pour une durée de $250 \mathrm{fs}$. Il ressort de ces différentes mesures que les paramètres optimaux sont :une impulsion de durée $250 \mathrm{fs}$ de $60 \mathrm{~mJ}$ à $800 \mathrm{~nm}$ focalisé dans un jet de xénon à 30 bars. Ce sont ces paramètres que l'on utilise pour les mesures présentées cidessous. 


\subsection{Mesure du spectre de photoélectron avec les paramètres optimisés}

Les spectres de référence (fig. 1 et 2) réalisés avec un canon à électron d'énergie $3 \mathrm{keV}$ permettent de déterminer les pics les plus importants, après nettoyage des cibles par bombardement ionique à l'argon. Les pics du cuivre à $62 \mathrm{eV}$ (transition $\mathrm{M}_{3} \mathrm{VV}$ ), et $918 \mathrm{eV}$ (transition $\mathrm{L}_{3} \mathrm{M}_{45} \mathrm{M}_{45}$ ) sont apparus comme les meilleurs candidats (résolution de $0.25 \mathrm{eV}$ ). Le premier pic est le plus intense donc le plus facile à étudier, et le second est de largeur adéquate ( $1.5 \mathrm{eV}$ à mi hauteur) pour les expériences de mesure de durée d'impulsion.

Les résultats obtenus avec les rayons x pour la raie à $62 \mathrm{eV}$ sont présentés sur les figures 3 et 4 . On observe un signal entre 55.5 et $64.5 \mathrm{eV}$ (précision $\pm 0.5 \mathrm{eV}$ ). Comparée au spectre de référence le nombre d'électron obtenu est bien moindre malgré une accumulation sur plus de 150000 tirs lasers, la probabilité de détecter un électron Auger étant inférieure à 1 électron par tir laser les fluctuations du signal sont non négligeables. Cependant l'accord obtenu sur la largeur du pic (ainsi que la position du pic) environ $10 \mathrm{eV}$ dans les deux cas est satisfaisant bien que la résolution soit différente entre les deux types de spectre. Ces éléments nous permettent de conclure que le signal observé est bien le pic d'électron Auger de la transition MVV.
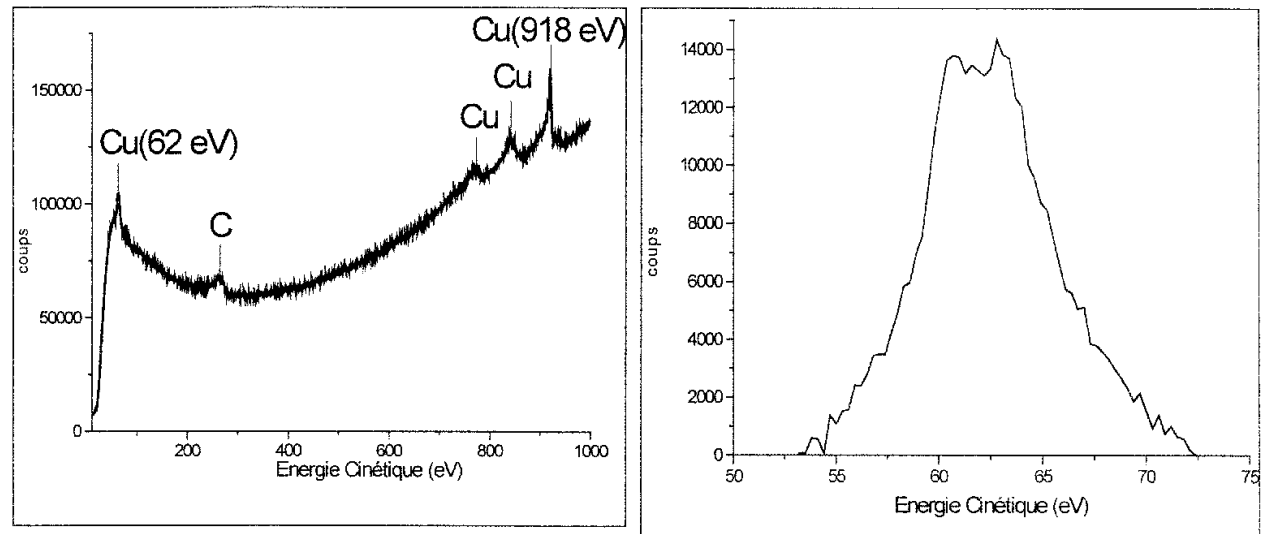

Figure 1. Spectre d'électron obtenu ave un canon à électron $(\mathrm{E}=3 \mathrm{keV})$.

Figure 2. Pic à $62 \mathrm{eV}$ du cuivre (transition MVV) obtenu avec le canon à électron (signal d'électrons

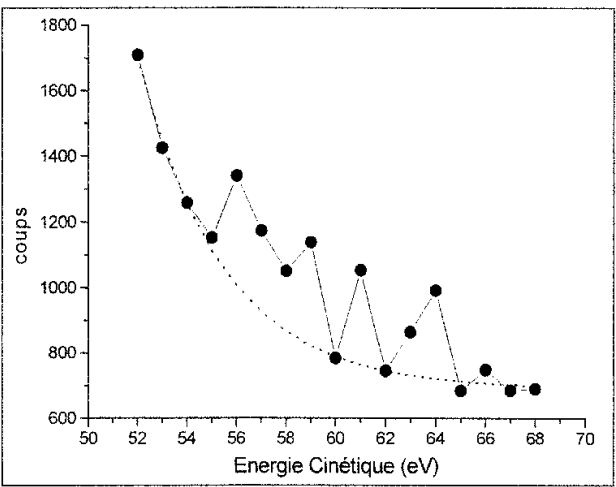
secondaires soustrait). Largeur à mi-hauteur de $10 \mathrm{eV}$.

figure 3 : spectre de photoélectron et interpolation du (2)

figure 4: Signal de photoélectron une fois le bruit bruit de fond (courbe pointillée). Accumulation de 50 soustrait et lissé (moyenne glissante sur 5 points) spectres avec $0.2 \mathrm{~s}$ d'acquisition par point 
Les résultats obtenus pour le pic de $918 \mathrm{eV}$ sont moins bons. Les spectres montrent bien la présence de signal mais malheureusement trop faible $(S / B \approx 2$ comparé à $S / B \approx 50$ pour le pic à $62 \mathrm{eV}$ ) pour pouvoir distinguer le pic Auger.

\section{DISCUSSION ET CONCLUSION}

Les résultats montrent clairement que le meilleur signal de photoémission est obtenu avec un jet de 30 bars de xénon irradié par des impulsions de 250 fs à $800 \mathrm{~nm}$ d'éclairement d'environ $2.10^{16} \mathrm{~W} / \mathrm{cm}^{2}$. De plus il est possible d'observer une raie Auger. Cependant le pic que nous observons est trop large pour mener à bien les expériences de mesure de durée d'impulsion. Le pic principal et les pics latéraux devraient se recouvrir et donc être indifférenciables. On pourrait au mieux dans ce cas détecter un élargissement de la raie. Un pic suffisamment étroit, selon le spectre de référence, serait celui à $918 \mathrm{eV}$. Dans ce cas nous devrions observer des pics latéraux bien distincts permettant une mesure de la durée d'impulsion. Le pic à $918 \mathrm{eV}$ n'est malheureusement pas discernable dans nos premiers spectres. La configuration actuelle de notre expérience est cependant largement améliorable afin d'obtenir plus de signal. Des améliorations sont en cours de réalisation sur notre expérience, comme l'ajout d'une optique $\mathrm{X}$ de focalisation.

La seconde étape de nos expériences consistera à sélectionner en longueur d'onde le rayonnement $\mathrm{X}$ avant de mesurer l'évolution temporelle d'une raie donnée. Les perspectives qui motivent ces recherches sont nombreuses. En effet l'utilisation de telles sources dans des expériences de type pompe-sonde sera d'un grand intérêt en physique des plasmas ou du solide : on peut penser à des expériences étudiant la dynamique de désorption des couches atomiques lors de l'ablation par laser de cibles solides.

\section{References}

[1] Ditmire T., Donnely T., Rubenchick A.M., Falcone R.W., Perry M.D., Phys. Rev. A 53(1996) 3379-3402

[2] Rozet J.P., Cornille M., Dobosz S., Dubeau J., Gauthier J.C., Jacquemot S., Lamour E., Lezius M., Normand D., Schmidt M., Vernhet D. Phys. Scrip. 92(2001)113-118

[3] Ditmire T., Tisch J.W.G.,Springate E., Mason M.B., Hay N., Smith R.A., Marangos J., Hutchinson M.H.R., Nature 386(1997) 54-56

[4] Shai Y.L., Phys. Rev. Let. 77(1996) 3343-3346

[5] Larsson J., Sjögren A., Rev. Scient. Instr. 70(1999) 2253-2256

[6] Schins J.M., Bréger P., Agostini P., Constantinescu R.C., Muller H.G., Grillon G.,

Antonetti A., Mysyrowicz A., Phys. Rev. Let. 73(1994) 2180-2183

[7] P.Auger, CR Acad. Sci. 177(1923)169 ; Surf. Sci. (NL) 48 (1975) 1-8

[8] Schins J.M., Bréger P., Agostini P., Constantinescu R.C., Muller H.G., Grillon G.,

Antonetti A., Mysyrowicz A., Phys. Rev.A 52(1995) 1272-1278

[9] Prigent C.,lamour E., Rozet J.P., Verhnet D.,Adoui L., Indelicato P., Gobert O.,

Meynadier P., Normand D., Perdrix M., J. Phys. IV, dans le même volume.

[10] Parra E., Alexeev I., Fan J., Kim K.Y., McNaught S.J., Milchberg H.M., Phys Rev. E 62(2000) R5931-R5934 